\title{
THE USE OF OXYGENATED WATER AND LYMPHATIC THERAPY IN THE TREATMENT OF POST-INJECTION PURULENT COMPLICATIONS IN DRUG ADDICTS
}

\author{
Vladimir Melnikov ${ }^{凶}(\mathbb{C}$, Arthur Bekbaev (i) \\ Astrakhan State Medical University, Astrakhan, Russia \\ mvvastr@mail.ru
}

\begin{abstract}
We compared the results of treatment and multifunctional examination of 78 patients (study group) with parenteral drug addiction and post-injection purulent lesions of the soft tissues and vessels of the lower limbs. In the study group (47), we used oxygenated drugs (OxyEnergya) and indirect lymphotropic administration of drugs. Systemic oxidative therapy was carried out by oral administration of OxyEnergya $15 \mathrm{ml}$ twice a day. In the comparison group (31), patients received conventional treatment. In the study group, the proposed complex treatment algorithm provides a relatively earlier (3-7 days) onset of the clinical effect of treatment.
\end{abstract}

KEYWORDS — drug addiction, phlegmon, phlebitis, oxygenated water, lymphatic therapy.

\section{| NTRODUCTION}

In recent years, the contingent of patients with parenteral drug addiction has changed, which is associated with the intravenous administration of synthetic drug substitutes [1]. After short-term or 1-2-fold intravenous drug administration, postinjection phlebitis develops, which is often combined with paravasal purulent-inflammatory infiltrates or phlegmon [2]. In most cases, purulent foci are localized in a functionally dangerous zone - the ileo-inguinal region and are characterized by extensive lesions and a tendency to spread along the great vessels [].Local post-injection complications are often accompanied by the development of gross changes in the homeostasis system. The success of treatment will be determined by the effectiveness of the antimicrobial and local treatment of purulent wounds, elimination of dysfunction of regulatory and effector systems organs [3].

The aim of the study is to prove the appropriateness of using oxygenated water and indirect lymphotropic combined drug therapy in the treatment of modern drug addicts with post-injection purulentinflammatory complications.

\section{MATERIALS AND METHODS}

In 2017-2019, 78 patients were treated with parenteral drug addiction with purulent-inflammatory post-injection lesions of the soft tissues and veins of the upper third of the right (55) and left (23) thighs. There were 49 men and 29women, aged 18 to 44 years, who injected surrogates of synthetic drugs (analogue of amphetamine) into the veins of the inguinal region. To determine the nature of post-injection complications, ultrasound of soft tissues and ultrasound Doppler scanning of the main veins of the lower limbs were performed. To improve the blood rheological properties and regional blood flow, low molecular weight dextrans (rheopolyglucin, reftan) were introduced. Purulent foci were opened under intravenous anesthesia. The lower limb was-dressed in Bellersplints.

In the study group (35), we carried out indirect lymphotropic administration of drugs. Inthe first interdigital space of the foot, ceftriaxone 1.0 diluted in $4 \mathrm{ml}$ of a $2 \%$ lidocaine solution +32 edlidase was injected strictly subcutaneously with the needle pointing to the dorsum of the foot, and after 5 minutes, $50 \mathrm{mg}$ of imunofan diluted in $5 \mathrm{ml}$ of isotonic solution of sodium chloride; fraxiparine in a dose of $0.6 \mathrm{ml}$ was injected more proximally. This manipulation was carried out 1 time per day with 48 hour intervals. Management of purulent wounds with oxygenated medicinal compositions (extempore: Levomekol ointment was mixed in 1:1 combination with OxyEnergya). Systemic oxidative therapy was carried out by oral administration of OxyEnergya by $15 \mathrm{ml}$ twice a day. OxyEnergya is a highly purified water enriched with oxygen molecules in an amount of $250,000 \mathrm{ppm} / \mathrm{l}$. OxyEnergya was given in the morning and evening peros in a volume of $30 \mathrm{ml}$.

In the comparison group (33), persons comparable by gender and age, had similar drugs administered by the traditional, intramuscular method, and their wounds were treated by dressings with Levomekol ointment.

The treatment results were evaluated clinically, by studying biochemical and laboratory blood parameters, bacteriological monitoring, including determination of microbial colonization of the wound wall, studying the cytokines profile, cytograms of wound contact preparation, $\mathrm{pH}$ measurement of wound 
exudate.The studies were performed upon admission of patiens, then on the $3-5^{\text {th }}, 7-9^{\text {th }}$ and $11-13^{\text {th }}$ days from the start of treatment.The data obtained were subjected to statistical processing by the STATISTICA 6.0 application software package. Indicatorswere considered reliable at $\mathrm{p}<0.05$ and $\mathrm{p}<0.01$.

\section{RESULTS, DISCUSSION}

In $61(78.2 \%)$ patients, phlebitis was accompanied by the formation of paravasalphlegmon, in 17 (21.8\%) - femoral soft tissue infiltration. Inflammation was characterized by severe clinical course, hyperthermia (up to $39^{\circ} \mathrm{C}$ ), leukocytosis (from 12.7 to $21.8 \cdot 10^{9} / \mathrm{L}$ ), blood LII up to 5.6. The presence of abscess was confirmed by ultrasound examination. Ultrasound scanning of the veins revealed parietal inflammation without valve insufficiency of the veins of the lower extremity. Ultrasound scanning of the veins revealed parietal inflammation without valve insufficiency of the veins of the lower limbs. At inoculation for flora, a mixt infection was detected: Staphylococcus aureus in combination with gram-negative flora. In the first group of patients, the general condition normalized at the $5-7^{\text {th }}$ day, the purulent process was localized within the damaged tissues. By the end of 7-9 days from the start of treatment, the cytological picture prevailed in the wound contact preparations, corresponding to the transition of the wound process to the phase of regeneration. By this period of treatment, the imbalance between pro- and anti-inflammatory cytokines was eliminated. On days 11-13, 17 patients had early secondary sutures; 5 had edges of their wound reduced with adhesive bandage strips. 13 patients had wound hiatus longer than $20 \mathrm{~cm}$ and it was closed in stages, starting from its corners (1-2 sutures) and so on for 3-5 days until the complete elimination of a wound defect. By the end of treatment,indicators of laboratory and biochemical blood tests were approaching the upper limit; LII was $1.8 \pm 0.2(\mathrm{p}<0.01)$. The average hospital stay was $17.8 \pm 2.2$ inpatient days.In the comparison group, the research results were significantly different, and the duration of treatment exceeded the one of the first group by 5-8 days.

In the study group, of the use of OxyEnergya and saturation of powerful collector of the inguinal lymphatic system with the antibiotic and immunomodulator contributes to a more rapid relief of local and general symptoms of purulent inflammation. Lymphatic therapy in combination with wound dressings saturated with oxygenated drug compositions allows targeted delivery of drugs to the lesion site [3, 4].

As a result, the terms of normalization of the general conditionand of elimination of homeostasis disorders are reduced by $1.5-2$ times, the symptoms of thrombophlebitis are more quickly relieved, the purulent process is localized within the damaged tissues, and wound microbial flora activity is suppressed [5]. As a result, the conditions are optimized for the passage of the purulent wound process into the regeneration phase, and the inpatient treatment time is reduced by $5.2 \pm 1.4$ inpatient days.

\section{CONCLUSIONS}

1. Post-injection purulent complications caused by synthetic surrogate drugs In modern patients with parenteral drug addiction are characterized by extensive damage of soft tissue. It proceeds according to the hyperergic type of inflammatory reaction with the development of an imbalance of pro- and anti-inflammatory cytokines.

2. Local and general use of OxyEnergya in combination with indirect lymphatic therapy improves the quality of care for this group of patients, and reduces the duration of inpatient treatment by an average of 5-7 days.

\section{REFERENCES}

1. BimalKanish, SandeepGoyal, AnuradhaBhaTIA. Cutaneous complications of infection drug abuse. Journal of Evolution of Medical and Dental Sciences 3(28):7755-7762. DOI: $10.14260 /$ jemds/2014/2967/

2. A.V. Sazhin, S.V. Lisin, D.Yu. Mikhailov. The structure of post-injection complications in patients with drug addiction in a surgical in-patient department. Russianmedicaljournal. 2012; 4: 17-19.

3. I.A. Yusupov, D.S. Parshin, S.S. Berebitsky. Endolymphatic antibiotic therapy of surgical diseases. Astrakhan: Publishing House of the Astrakhan State Medical Academy; 2005. Availableat: https://search. rsl.ru/ru/record/01002755379.

4. Suvarnapathaki, S., WU, X., Lantigua, D. eT AL. Breathing life into engineered tissues using oxygenreleasing biomaterials. NPG Asia Mater 11, 65 (2019). https://doi.org/10.1038/s41427-019-0166-2

5. G.V. Zhurbina, V.V. Melnikov, D.S. Parshin. Lymphatic therapy in the complex treatment of post-injection purulent complications in patients with parenteral drug addiction. Surgery. 2016; 2: 12-17. (2) 